\title{
Gender Dimorphism of Crania in Young Jordanian Adults: Discriminant Function Analysis Approach for Gender Prediction
}

\author{
Wala Aminn ${ }^{\mathrm{a}, \mathrm{b}}$, Dina Othman ${ }^{\mathrm{a}}$
}

\begin{abstract}
Background: Determination of gender using craniofacial characteristics, though made common worldwide, Jordan is still lacking of such investigation. The aim of this study was to develop a gender determination technique for young Jordanian adult population using osteometric data, from cephalometric images, and discriminant function analysis.
\end{abstract}

Methods: A total of 146 randomly selected digital lateral cephalometric radiographs of fully dentate young adult patients were used in the investigation; 47 patients were males and 99 were females. For each lateral cephalometric radiograph 19 craniofacial skeletal landmarks were digitized by one observer using a customized analysis created in Viewbox 4-Cephalometric Software subroutines. Utilizing the digitized landmarks, 18 measurements that comprised 14 linear, three angular and one proportional parameters were carried out.

Results: The results demonstrated that, with the exception of the Menton to Gonion distance, i.e. the length of the mandibular body, the mean values of all other parameters of male subjects were statistically significantly larger $(\mathrm{P}<0.05)$ than those for females. Mastoid height was found to be the best single predictor of gender and can provide an accuracy rate of $82.2 \%$. The stepwise method revealed four dimensions (mastoid height, mastoid width, glabella to supraglabellare-nasion distance, and the length of skull base) were found to form the best combination of parameters most precisely to depict the best possible prediction, raising the classification accuracy up to $87.7 \%$.

Conclusions: A discriminant function equation specific for Jordanian population has been derived from cranio-mandibular variables. The equation can now be used for a calculable and more precise prediction of gender of Jordanian young adult population.

Manuscript accepted for publication August 28, 2014

${ }^{a}$ Division of Clinical Dentistry, Jordan University Hospital, University of Jordan, Amman, Jordan

${ }^{b}$ Corresponding Author: Wala M. Amin, Faculty of Dentistry, University of Jordan, Amman 11942, Jordan. Email: wami@ju.edu.jo

doi: http://dx.doi.org/10.14740/jcs241w
Keywords: Sex determination; Mastoid process; Cranio-mandibular; Discriminant analysis; Craniometry

\section{Introduction}

Sex determination from skulls has conventionally been done using either visual assessment based on morphological traits presented by various bones of craniofacial structures or morphometric methods employing linear and/or angular dimensions. It has been reported that morphological characters such as supraorbital ridges, mastoid processes, cheek bones, orbit and mandible give valuable idea of gender $[1,2]$. In terms of strength of prominence of these characters, the female skull inclines for lower values compared to those demonstrated by male skull. Some researchers [3] employed the petrous bone in sexual dimorphism and reported that $74 \%$ accuracy was attained. Others [4] studied flexure along the posterior border of the mandible as morphologic indicator of sexual dimorphism and claimed a total accuracy of $94.2 \%$ was reached. Steyn and Iscan [5] examined cranium, vault, face, mandible and bizygomatic width and claimed that all these gave differentiation values above $80 \%$. Supraorbital margin, glabella, mastoid process, crista supramastoid and mandible were employed in sexual dimorphism, and claimed to yield reliable results related to sex reaching $70-91 \%$ accuracy $[6,7]$. Analyzing zygomatic extension and malar size for skull dimorphism, Roger [8] reported that $88 \%$ accuracy was achieved. Other cranio-mandibular parameters namely, mastoid size, supraorbital ridge, zygomatic extension, nasal aperture, and gonial angle were assessed for sex determination [9], and these morphological features were reported to performed well and were identified as high-quality traits. $\mathrm{Hu}$ et al [10] stated that "rocker-shaped contour of mandibular lower border predominated in males whereas female mandibles were mostly found straight"; it was also reported that the chin in most men was found generally square but that of females was found more pointed. It was commented that the strength of such parameters depended upon physical activity and puberty state. Mandible length, ramus height and bi-gonion breadth measurements were found to be sexu- 


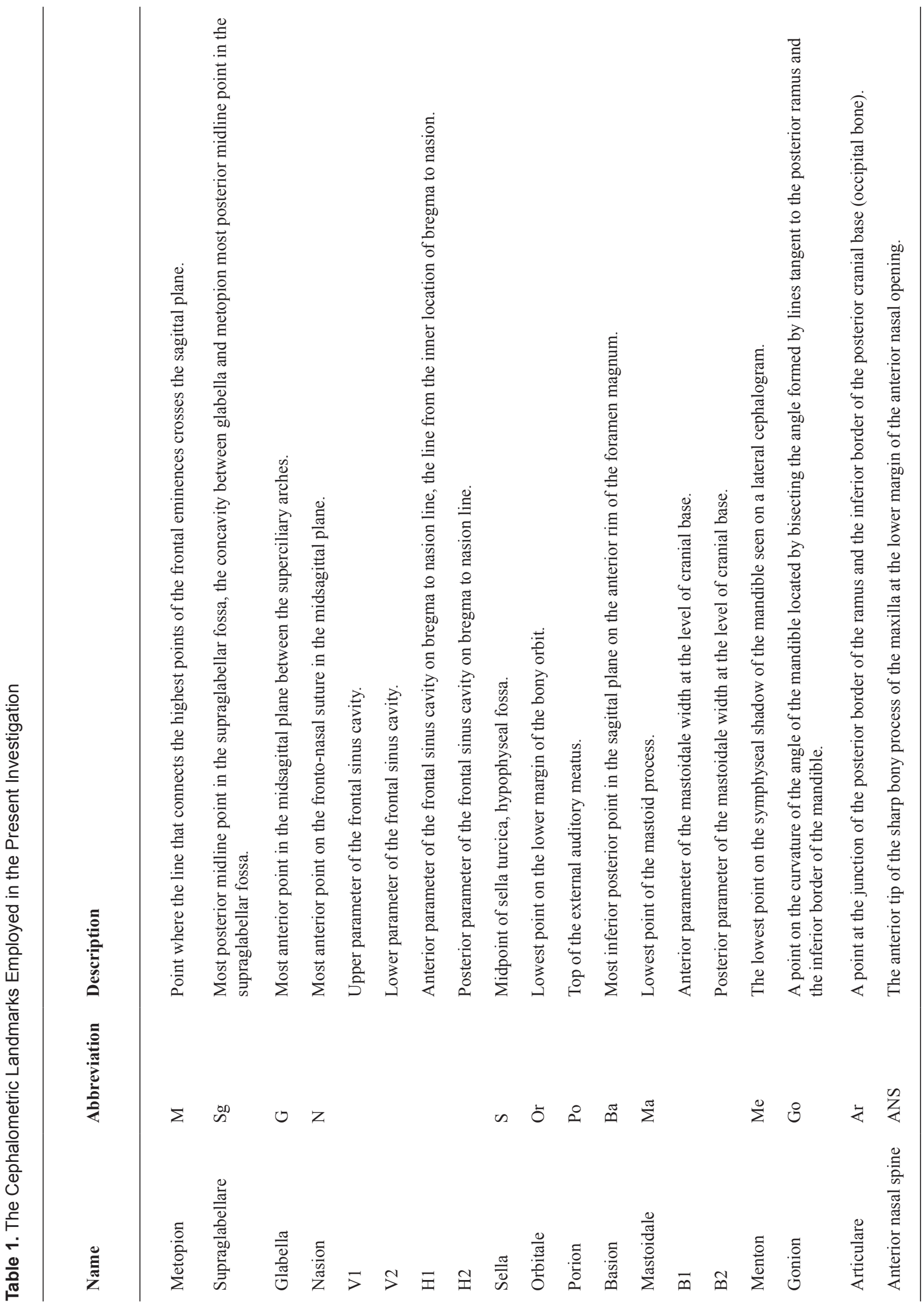




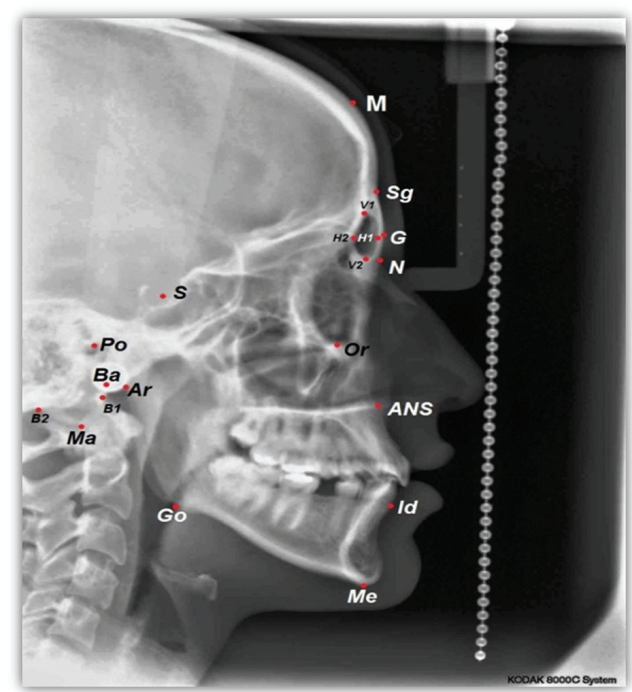

Figure 1. A cephalometric radiograph illustrating the landmarks defined in Table 1.

ally dimorphic in black South Africans [11]. Kranioti et al [12] carried out osteometric measurements on cranio-facial skeletons on the bizygomatic breadth, cranial length, nasionprosthion and mastoid height, and nasal breadth of 90 males and 88 females. Their results indicated that males were statistically significantly greater than females in all dimensions. Using cephalometric sexual dimorphism, it was found [13] that angular measurements made on those images were not of significant dimorphic features. Females' cranial dimensions were found smaller than those of males [13]. Cephalograms of 100 equally distributed males and females were analyzed [14] and the radius of glabellar eminence curvature and that of nasal root were assessed as dimorphic feature. Males showed significantly smaller values than females which led to the conclusion that glabellar eminence had most differentiating feature with an accuracy of more than $90 \%$. The glabellar projection index (GPI) together with the frontal sinus width (FSWd) and a linear measurement of inionopisthocranion to basion-nasion (IOpBaN) were analyzed on 100 cephalograms of equal male and female Korean subject and showed differentiation accuracy of $98 \%[15,16]$. Linear measurements of mandibular dimensions of males and females included bigonial width, bicondylar breadth, mandibular symphasis height, mandibular length, and mandibular ramus height, which were reported to have significant sexual dimorphism [17]. The role of mastoid process as sexual dimorphism feature was evaluated [18], in which the results of measurements of a triangular area that covered the mastoid process indicated that total area more than $1,447.4 \mathrm{~mm}^{2}$ denoted male pattern whereas area less than $1,260.4 \mathrm{~mm}^{2}$ showed female predilection. Patil and Modi [19] assessed 10 craniofacial parameters for sex identification, of which seven parameters were found most reliable attaining $99 \%$ ac-

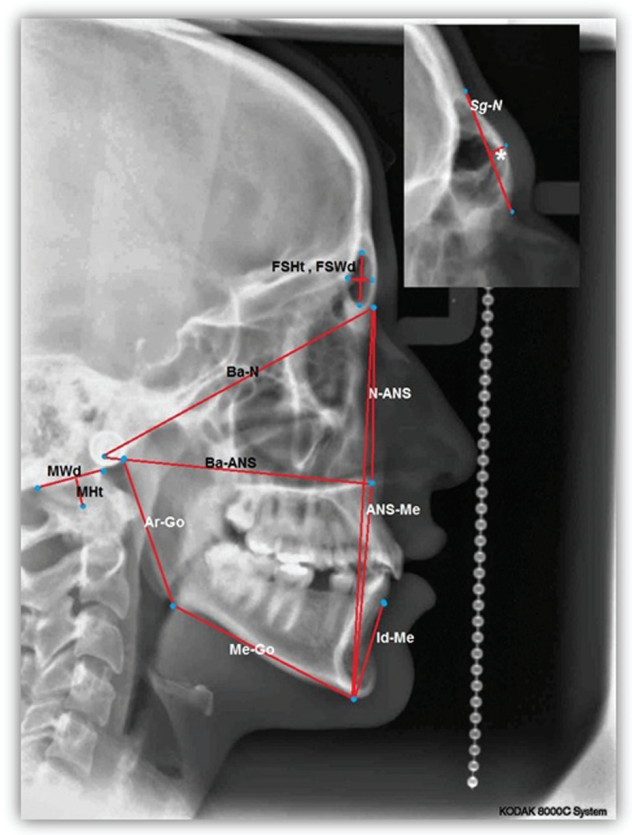

Figure 2. A cephalometric radiograph illustrating the linear measurements defined in Table 2. *GSg-N.

curacy. They were related to length of base of skull, height and width of mastoid process, depth and height of face, and maximum cranial length. In a separate study Veyre-Goulet et al [20] examined 114 lateral cephalograms of European subjects; eight variables were evaluated for sexual dimorphism, six of which were related to the anterior region, gave a 95.6\% accuracy, and thus considered reliable gender identifiers. However, it was reported that they could be subject to ethnic variations.

\section{The present study}

The present investigation aimed, firstly, to trace and measure a number of selected cranio-mandibular parameters on lateral cephalograms of Jordanian subjects, using "Viewbox" orthodontic software, and secondly, to assess the reliability of those parameters in determining the gender of the study subjects with the help of discriminant function analysis.

\section{Materials and Methods}

The present study was carried out on a representative sample comprising 146 randomly selected digital lateral cephalometric radiographs of fully dentate young adult patients, who attended the Clinics of the Dental Department of the Jordan University Hospital (JUH). The average age of the patients who formed the sample was 20 years and ranged between 13 and 27 years. The lower limit of the age range was set at or above the age of puberty based on Krogman's propo- 


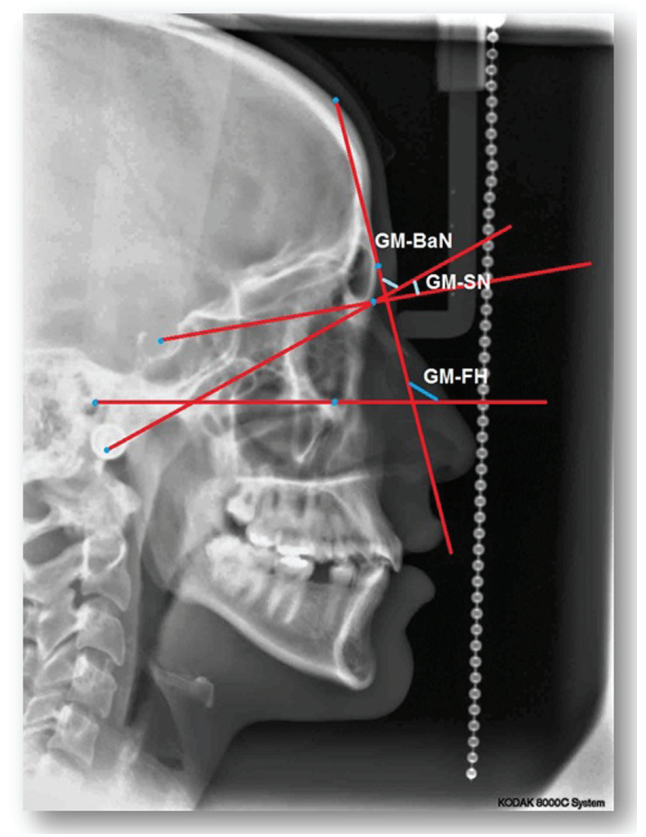

Figure 3. A cephalometric radiograph illustrating the angular measurements defined in Table 2.

sition which stated that "the cranio-mandibular parameters are age phenomena appearing or becoming pronounced at puberty, hence sex determination below this age range may show high variation" [1]. Of the total sample, 47 patients were males and 99 were females. The exclusion criteria used in selecting the test sample involved excluding patients with a history of previous orthodontic or orthognathic treatment, OPT images that showed artifacts preventing proper landmark determination and images of poor resolution.

Prior to conducting the investigation, and in compliance with the policy of the Clinical Research Authority at the JUH, signed written informed consents were obtained from all the subjects selected for the study. All subjects were made aware that their lateral cephalogram images were included in the investigation. The experimental protocol was examined and approved by the Ethics Committee and was, therefore, performed in accordance with the ethical standards laid down in the 1964 Declaration of Helsinki, last edited in 2013 [21].

The Dental Department at the JUH uses a computerized Kodak Dental Imaging system (Kodak Laboratories, Rochester, NY, USA), the software of which was designed to process lateral cephalometric radiograph images and saves them as "Joint Photographic Experts Group"(JPEG) file format.

For each lateral cephalometric radiograph 19 craniofacial skeletal landmarks were digitized (Table 1 and Fig. 1) by one observer using a customized analysis created in Viewbox 4-Cephalometric Software (dHAL Software, Kifissia, Greece). Utilizing the selected and digitized landmarks, measurements that comprised 14 linear (Fig. 2), three angu- lar (Fig. 3) and one proportional parameters were carried out (Table 2).

In order to eliminate the possibility of inter-observer variations which, sometimes, arise in similar studies, measurements of all parameters of the entire sample were carried out by the same observer (DAO).

\section{Statistical treatment}

All 18 measurements from each radiograph processed by Viewbox 4-Cephalometric Software were transferred and organized according to gender that was represented by the binary variables, " 1 " for males and " 2 " for females. Of the entire sample, cephalograms of 10 randomly selected subjects were analyzed twice, separated by 2 weeks, by the same observer. Data sets of the two observations were statistically treated using a paired $t$-test to check for intra-observer variation. Statistical analysis was conducted using the Statistical Package for the Social Sciences (SPSS) version 15.0. Data analysis was carried out by canonical discriminant function analysis to find linear combinations of those parameters that best separate the two genders. In order to select the combination of parameters that best discriminate the two genders, stepwise discriminant function analysis was used (utilizing the Wilks lambda method). A leave one out classification procedure was applied to demonstrate the accuracy rate of the original sample as well as that which resulted by crossvalidation, and this method was used to reduce the overestimate of the predictive values.

\section{Results}

The results of the paired $t$-test that was carried out to check the intra-observer variations indicated no statistically significant difference between measurements $(\mathrm{P}>0.05)$ recorded at two different occasions $(t=0.932$ for males and $t=0.937$ for females). The descriptive statistics of all parameters of the sample indicated significant difference between sexes ( $P$ $<0.05$ ) except for two parameters, namely, the Me-Go and FSWd (Table 3). When the discriminant function analysis compares between two groups, as in the present investigation whereby the comparison was made between males and female, the value of the univariate F, presented in Table 3, equals to the squared " $t$ " value. The results demonstrated that, with exception of the "Me-Go" parameter, the means of all other parameters for male subjects were larger than those for females (Table 3 ).

In this model, the mastoid height "MHt" was found to be the best single predictor of gender with a prediction accuracy of $82.2 \%$. Whereas, four parameters, the mastoid height "MHt", the mastoid width "MWd", the glabella and the supraglabellare to nasion line distance "GSg-N" and the basion to nasion distance "Ba-N" were found to form the 
Table 2. The Cephalometric Parameters Employed in the Present Study

Parameter

\section{Description}

Linear measurements

Ba-ANS

$\mathrm{Ba}-\mathrm{N}$

N-ANS

ANS-Me

$\mathrm{N}-\mathrm{Me}$

Id-Me

Ar-Go

Me-Go

FSHt

FSWd

$\mathrm{MHt}$

MWd

Sg-N

GSg-N

Angular measurements

GM-Ba-N

GM-SN

GM-FH

Proportional

GPI
Basion to anterior nasal spine.

Basion to nasion.

Nasion to anterior nasal spine.

Anterior nasal spine to Menton.

Nasion to Menton.

Infradentale to Menton.

Articular to gonion.

Menton to gonion.

V1 to V2.

$\mathrm{H} 1$ to $\mathrm{H} 2$.

Mastoidale to B1-B2.

$\mathrm{B} 1$ to $\mathrm{B} 2$.

Supraglabellar to nasion.

Glabella to Sg-N (distance between glabella and the supraglabellare to nasion line).

Glabella-metopion to Basion-nasion.

Glabella-metopion to Sella-nasion.

Glabella-metopion to Frankfort plane (porion-orbitale).
Depth of face.

Length of skull base.

Height of upper face.

Height of lower face.

Anterior height of face.

Height of mandibular symphasis.

Height of mandibular ramus.

Length of mandibular body.

Frontal sinus height.

Frontal sinus width.

Mastoid height.

Mastoid width. best combination of parameters most precisely to depict the best possible prediction. The contribution fraction of the four discriminant parameters to gender prediction is illustrated in Fig. 4.

The discriminant function predictive equation derived from the coefficients of the four best predictors selected by the stepwise analysis (Table 4) was:

$$
\mathrm{DF}=0.6904 \times \mathrm{MHt}+0.4378 \times \mathrm{MWd}+0.3789 \times \mathrm{Ba}-\mathrm{N}
$$

$+0.3306 \times \mathrm{GSg}-\mathrm{N}$

The group centroid discriminant score for males was equal to 1.297 and for females was -0.616 , as indicated by the discriminant analysis. The sectioning point was equal to 0.3405 , which was calculated by following equation:

Cut score $=(1.297+(-0.616)) / 2=0.3405$

Accordingly, when a DF score is above the section- ing point, the subject being investigated is probably a male whereas, scores below that are likely to indicate female subjects.

Values of the predictive accuracies resulted from direct discriminant analysis and leave one out classification in different models, where combinations of parameters at a time were used; all parameters, angular, linear, proportional, and stepwise resulted models are summarized in Table 5.

Values of the predictive accuracy of the different models (Table 5) showed that two models, namely the all parameters and the all linear measurement parameter demonstrated the same overall cross-validated sexing accuracy $(85.6 \%)$. This accuracy was found only marginally improved by the stepwise method $(87.0 \%)$ which affected a small increase in the overall sexing accuracy of $1.61 \%$. The all angular mea- 


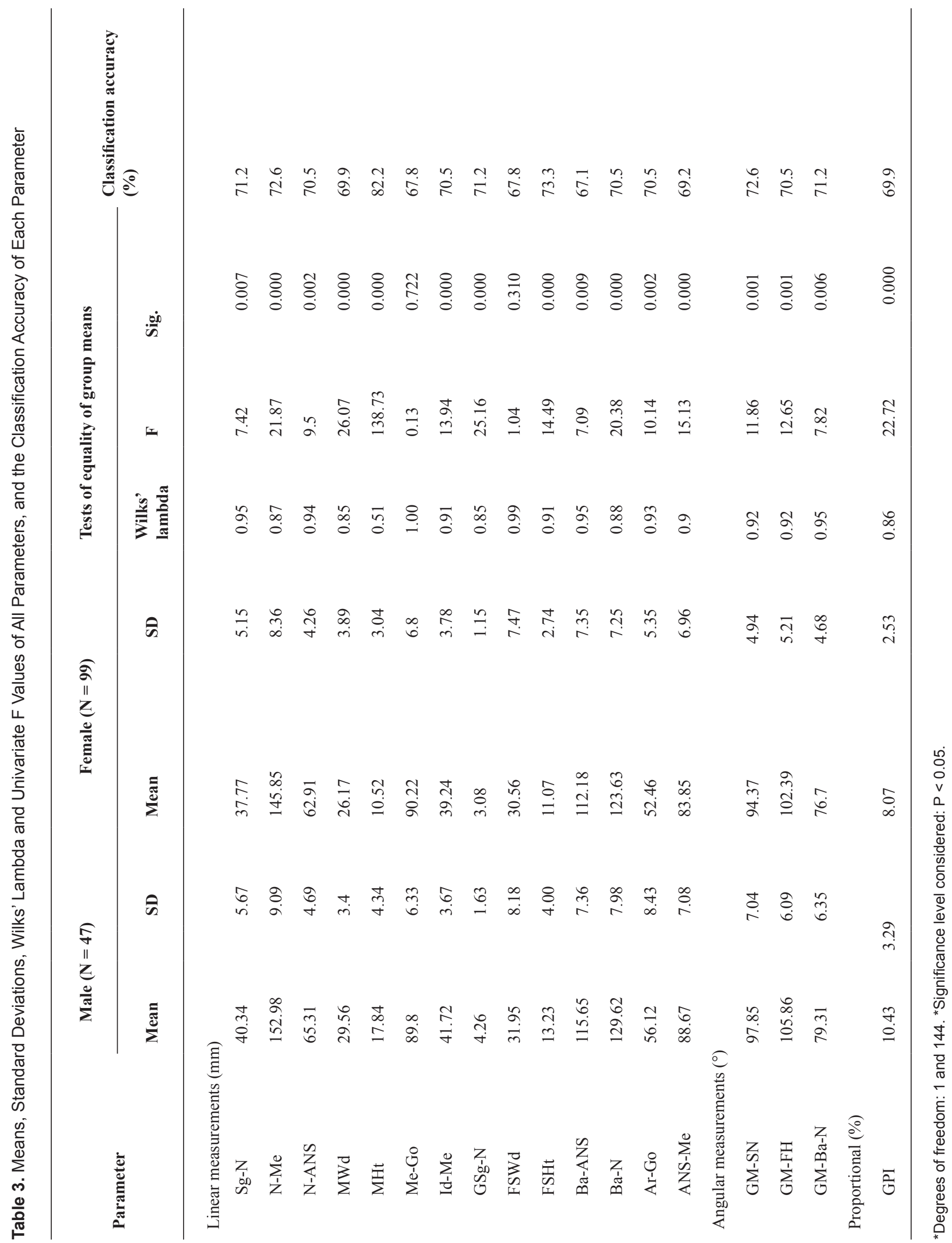




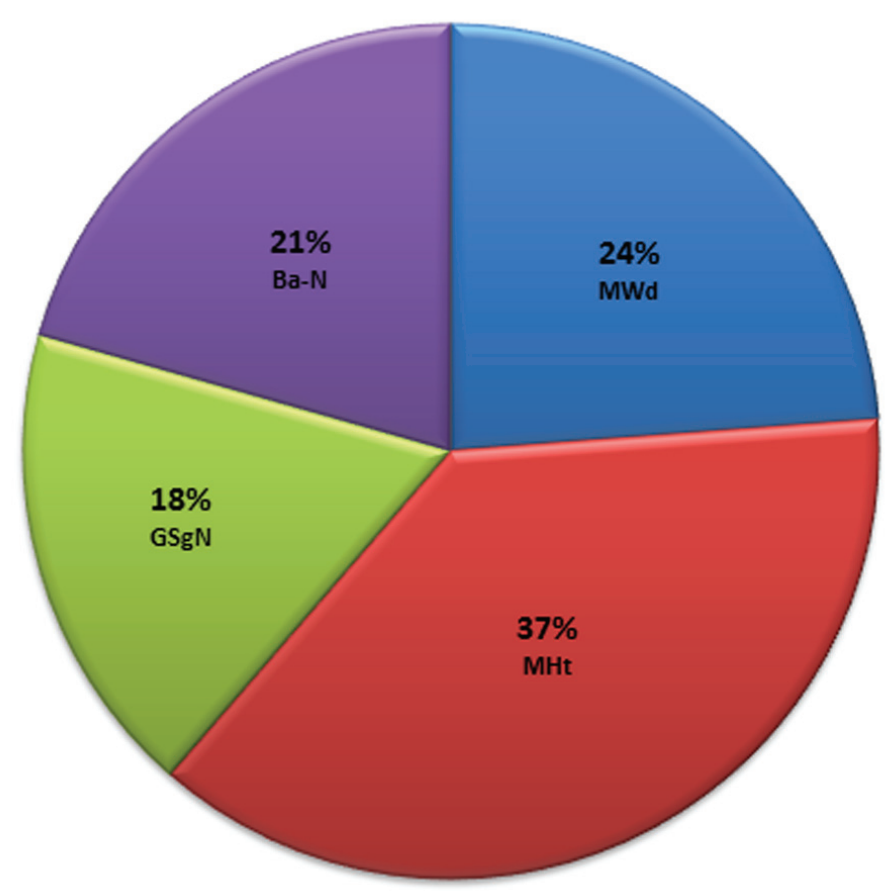

Figure 4. The contribution fraction of the discriminant parameters to gender determination.

surement parameter as well as the proportional parameter models both demonstrated lower overall sexing accuracies after cross-validation, with the former parameter showing a $70.5 \%$ and the latter showing a $69.9 \%$.

Despite the variations among models in the value of the overall cross-validated accuracy, they all demonstrated a sex bias accuracy towards the female group. The different models varied in the degree of bias that they demonstrated in favor of the female group, with the all parameters as well as the all linear parameters, the angular, the proportional, and the stepwise method models showing bias of $21.3 \%, 77.3 \%$, $69.2 \%$ and $23.0 \%$, respectively.

\section{Discussion}

Gender identification of humans from skeletal remains or their radiographic images can be of a high accuracy if a complete skeleton was available for analysis. Since availability of an intact skeleton is unlikely, it becomes essential that a set of identifiers or predictors for each bone to be developed utilizing the morphological variables of each bone [8]. Different methods of evaluation of skull bones for dimorphism were reported to the literature. These involved a method of visual assessment of morphology which is condemned of being a highly subjective method, thus, could not be employed

Table 4. The Outcome of the Stepwise Discriminant Analysis Indicating the Parameters and Their Corresponding Standardized Coefficients Which Entered the Discriminant Equation

Parameter

$\mathrm{MHt}$

GSg-N

Ba-N

\section{Coefficient}

0.4378

0.6904

0.3306

0.3789 


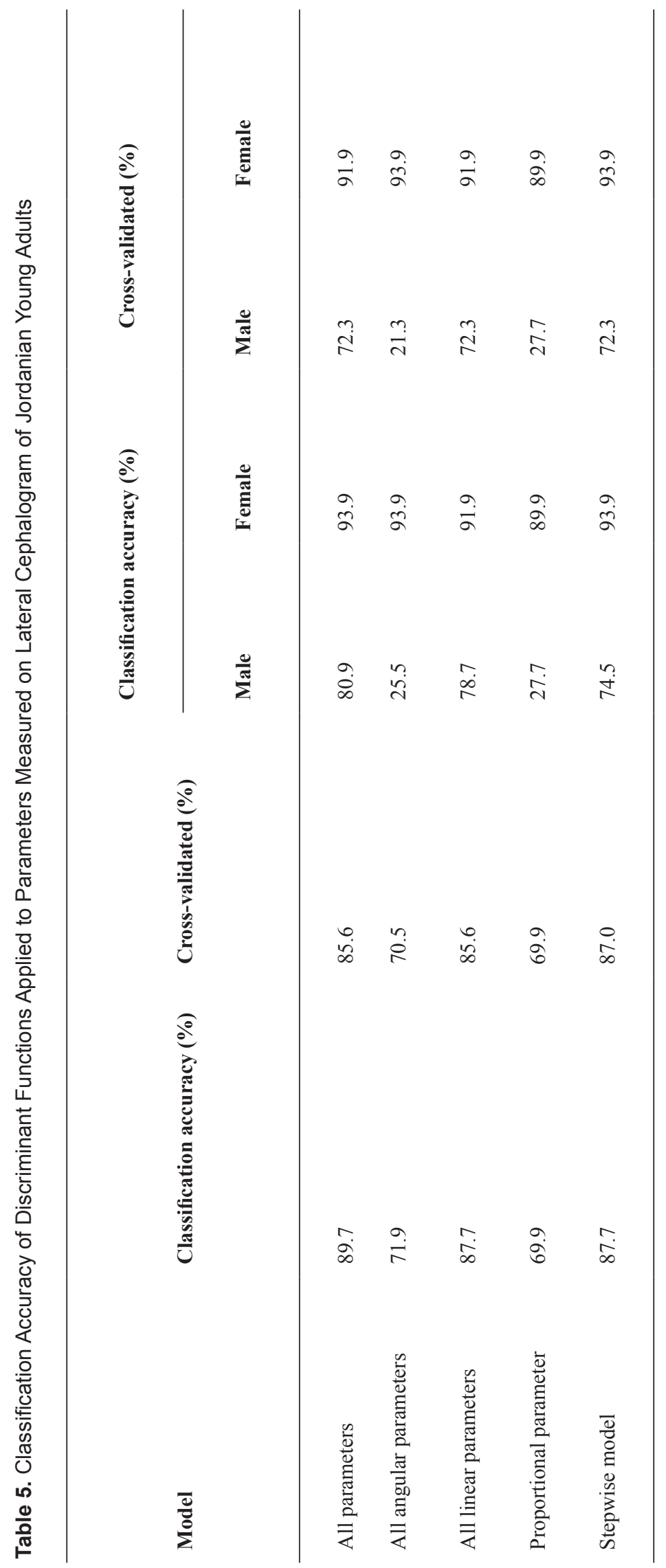


in investigations conducted by more than one examiner due to inter-observer variations. In addition, the method may not be suitable for studies that involve cosmopolitan societies because of the considerable inter-population or ethnic and inter-racial differences in the skeletal bones morphology.

More accurate methods that depend on measurements and morphometry like cephalometry were advocated for use in determination of gender from skulls [19]. Despite the fact that morphometric traits are more objective, a random set of measurements may not invariably be conclusive but such data require rigorous statistical treatment using an appropriate technique. The most suitable and widely used statistical tool has been multivariate discriminant analysis of sex determination for skeletal measurements. The advantage of using the discriminant function analysis in association with the morphometric trait measurements is that this method of analysis classifies individuals into two or more alternative groups, and in the present study the subjects were classified into male and female groups, on the bases of set measurements [15]. The method identifies which variables contribute to making the classification, thus it serves as an entirely objective statistical technique for sex determination [15]. It has been emphasized that combining the two approaches, i.e. the morphometric trait measurement and the discriminant function analysis improves accuracy as the two methods complement each other [22].

Lateral cephalometric radiographic images of the study subjects were used as they are more objective, standardized and reproducible [20]. This radiographic method describes the three-dimensional characters of the skull on a roentgenogram that presents a two-dimensional image. Thus the morphometric traits of the cranio-mandibular superstructures and intracranial structures can be easily assessed [15].

It has been reported that the most indicative regions of the skull in terms of sexual dimorphism are the frontal regions and base of the skull which are likely to be preserved during mass disasters $[1,15,20]$. Accordingly, the present investigation involved cranio-mandibular traits, and all measurements made on lateral cephalometric images of our Jordanian sample. The 18 variables that were analyzed in the present study were equal to the number of variables employed in two previous reports $[15,20]$ and more than those adopted by Patil and Mody [19] who evaluated 10 variables, and more than those used by Barthelemy et al [17] who analyzed only seven variables. Obviously, the more the number of the investigated variables means more elaborate analyses are conducted which give rise to more precise and accurate outcomes.

Univariate descriptive statistics showed all the parameters except the length of the body of the mandible (Me-Go) contributed in determination of sex. The mastoid height (MHt) was found to be the best single predictor of gender with a prediction accuracy of $82.2 \%$. The mastoid height $(\mathrm{MHt})$, in addition to the mastoid width (MWd), the distance from glabella and the midpoint between supraglabellare to nasion (GSg-N), and the length of skull base (Ba-N) entered the discriminant function predictive equation of the Jordanian population.

The results showed that the mean values of all parameters except the FSWd and the Me-Go were higher in males compared to females in the range of $3.0-41.03 \%$. These findings supported those reported by other investigators; Bibby [13] found that male skulls are $8.5 \%$ larger than female skulls; other researchers [20,23] also showed that the linear dimensions of male skulls were generally greater than the corresponding measurements in female skulls. Hsiao et al [15] reported that the mean male linear dimensions as well as proportional measurements were greater than those in females.

Mastoid height entered the discriminant predictive function and was a significant factor in sexual dimorphism. Several studies have shown that mastoid height is a consistently reliable parameter in both morphometric and cephalometric studies irrespective of the population evaluated $[1,5,8,9$, $15,16]$. An increase in mastoid height reflects growth at the base of the skull which affects an increase in the size of the mastoid bony process. Extended growth in male gives rise to an increase in mastoid size. Moreover, the relatively more vigorous musculature in males than in females, particularly the stronger sternocleidomastoids may contribute to the downwards prolapse of the male mastoids more than the corresponding structures in the female. This explains why males have definitely larger mastoid height and more robust skull.

In this study the parameter that measured the length of skull base or cranium (Ba-N) entered the specific discriminant function which correctly sexed $87.0 \%$ of young Jordanians.

The overall cross-validated accuracy for the Jordanian population was $85.6 \%$ which was improved to $87.0 \%$ by the stepwise method. This is comparable with the accuracy of $87.39 \%$ reported by Barthelemy et al [17] in their survey on South-West France population using mandibular dimorphism. However, the accuracy arrived at in this investigation was less than the $100 \%$ accuracy reported by Hsiao et al [15] who carried out their investigation on Taiwanese population using lateral cephalograms of cranial traits. The reason for the lesser accuracy may be ascribed to the use of different cephalometric parameters in the determination of gender. In general, the differences in the overall accuracy reported by different researchers could be attributed to the fact that the discriminant function equations are population specific and probably sensitive to ethnic and racial variations.

\section{Conclusions}

A specific discriminant function was created for young Jordanian adults from 18 established cephalometric craniomandibular variables. A total of 146 cases were classified into 
two sexual groups with $87 \%$ accuracy. Among the 18 variables, the mastoid height "MHt" alone showed the greatest efficiency as a single discriminator, with $82.2 \%$ accuracy. It was possible to determine the sex of the sample with $73.5 \%$ accuracy with four variables (MHt, MWd, Ba-N, and GSg$\mathrm{N})$. With the exception of the frontal sinus width and the mandibular body length, the mean values of all parameters were $3-41 \%$ higher in males than in females.

\section{Disclosures}

The authors declare they have neither financial disclosure nor conflict of interest.

\section{References}

1. Krogman WM. The human skeleton in forensic medicine. Springfield. Illinois, USA. Charles C. Thomas Pub Ltd. 1962;114-122.

2. Biggerstaff RH. Craniofacial characteristics as determinants of age, sex, and race in forensic dentistry. Dent Clin North Am. 1977;21(1):85-97.

3. Kalmey JK, Rathbun TA. Sex determination by discriminant function analysis of the petrous portion of the temporal bone. J Forensic Sci. 1996;41(5):865-867.

4. Loth SR, Henneberg M. Mandibular ramus flexure: a new morphologic indicator of sexual dimorphism in the human skeleton. Am J Phys Anthropol. 1996;99(3):473485.

5. Steyn M, Iscan MY. Sexual dimorphism in the crania and mandibles of South African whites. Forensic Sci Int. 1998;98(1-2):9-16.

6. Graw M, Czarnetzki A, Haffner HT. The form of the supraorbital margin as a criterion in identification of sex from the skull: investigations based on modern human skulls. Am J Phys Anthropol. 1999;108(1):91-96.

7. Graw M. Significance of the classical morphological criteria for identifying gender using recent skulls. Forensic Sci Communications. 2001;3(1):1-8.

8. Rogers TL. Determining the sex of human remains through cranial morphology. J Forensic Sci. 2005;50(3):493-500.

9. Williams BA, Rogers T. Evaluating the accuracy and precision of cranial morphological traits for sex determination. J Forensic Sci. 2006;51(4):729-735.

10. Hu KS, Koh KS, Han SH, Shin KJ, Kim HJ. Sex de- termination using nonmetric characteristics of the mandible in Koreans. J Forensic Sci. 2006;51(6):1376-1382.

11. Franklin D, O'Higgins P, Oxnard CE, Dadour I. Discriminant function sexing of the mandible of indigenous South Africans. Forensic Sci Int. 2008;179(1):84 e8185.

12. Kranioti EF, Iscan MY, Michalodimitrakis M. Craniometric analysis of the modern Cretan population. Forensic Sci Int. 2008;180(2-3):110 e111-115.

13. Bibby RE. A cephalometric study of sexual dimorphism. Am J Orthod. 1979;76(3):256-259.

14. Funayama M, Aoki Y, Kudo T, Sagisaka K. Sex determination of the human skull based upon line drawing from roentgen cephalograms. Tohoku J Exp Med. 1986;149(4):407-416.

15. Hsiao TH, Chang HP, Liu KM. Sex determination by discriminant function analysis of lateral radiographic cephalometry. J Forensic Sci. 1996;41(5):792-795.

16. Hsiao TH, Tsai SM, Chou ST, Pan JY, Tseng YC, Chang HP, Chen HS. Sex determination using discriminant function analysis in children and adolescents: a lateral cephalometric study. Int J Legal Med. 2010;124(2):155160.

17. Barthelemy I, Telmon N, Brugn JF, Rouge D, Larrouy G. Cephalometric study of mandibular dimorphism in living population in South-West France. International Journal of Anthropology. 1999;14(4):211-217.

18. Pavia LAS, Segre M. Sexing the human skull through the mastoid process. Rev Hop Clin Fac Med Sao Paulo. 2003;58(1):15-20.

19. Patil KR, Mody RN. Determination of sex by discriminant function analysis and stature by regression analysis: a lateral cephalometric study. Forensic Sci Int. 2005;147(2-3):175-180.

20. Veyre-Goulet SA, Mercier C, Robin O, Gurin C. Recent human sexual dimorphism study using cephalometric plots on lateral teleradiography and discriminant function analysis. J Forensic Sci. 2008;53(4):1-4.

21. World Medical Association, "WMA" Declaration of Helsinki-Ethical Principles for Medical Research Involving Human Subjects. Proceedings of the 18th General Assembly, Helsinki, Finland, Report; 1-8, June 1964.

22. Mahesh K, Moh ML, Patnaik VVG. Determination of sex by discriminant function analysis: Cephalometric study. Int J Pure App Biosci. 2013;1(4):18-21.

23. Ingerslev $\mathrm{CH}$, Solow B. Sex differences in craniofacial morphology. Acta Odontol Scand. 1975;33(2):85-94. 